\title{
Modelos experimentales en la cirugía laparoscópica urológica
}

\author{
Usón Gargallo J*, Sánchez Margallo FM*, Díaz-Güemes Martín-Portugués I*, \\ Loscertales Martín de Agar B*, Soria Gálvez F*, Pascual Sánchez-Gijón S.** \\ "Departamento de Laparoscopia. Centro de Cirugía de Mínima Invasión, Cáceres. ${ }^{* *}$ Servicio de Cirugía. \\ Hospital Santa María del Puerto, El Puerto de Santa María. Cádiz.
}

Actas Urol Esp 2006; 30 (5): 443-450

\section{RESUMEN}

MODELOS EXPERIMENTALES EN LA CIRUGÍA LAPAROSCÓPICA UROLÓGICA

Presentamos la experiencia del Centro de Cirugía de Mínima Invasión (CCMI) en el desarrollo de un modelo de entrenamiento secuencial para la formación en cirugía laparoscópica urológica dotado con diferentes niveles de experiencia. Este análisis incluye la descripción de los objetivos de formación, el proceso de enseñanza de las técnicas urológicas sencillas y avanzadas y algunas consideraciones actuales y futuras antes de aplicar con éxito las técnicas laparoscópicas. El resultado final del programa de aprendizaje pretende optimizar la formación del cirujano y la práctica clínica de estas técnicas quirúrgicas.

Las etapas del aprendizaje han sido clasificadas en cuatro niveles, diferenciándose a su vez una serie de módulos y modelos para el aprendizaje cuya aplicación dependerá de la experiencia y destreza del cirujano. Basado en este sistema piramidal de entrenamiento hemos establecido un programa de aprendizaje donde el alumno avanza paso a paso a través de cada nivel dependiendo de su nivel de habilidad.

Presentamos nuestra experiencia en doce cursos de formación en laparoscopia urológica y cuatro cursos de formación en prostatectomía radical laparoscópica, habiendo asistido más de 300 urólogos a dichas actividades. Por otra parte, destacamos la puesta en marcha conjunta con algunos Servicios de Urología españoles de programas especiales de aprendizaje experimental de técnicas más complejas, como la prostatectomía radical laparoscópica, nefrectomía parcial o pieloplastia desmembrada de Anderson-Hynes.

Como ya hemos advertido en otros trabajos, y como aseveran otros grupos, el aprendizaje laparoscópico por niveles constituye un concepto muy útil para evitar problemas derivados de un aprendizaje quirúrgico incompleto e incorrecto, al tiempo que permite reducir la curva de aprendizaje de las técnicas quirúrgicas urológicas haciendo posible implantar técnicas de cierta complejidad técnica en la práctica quirúrgica habitual en la especialidad de Urología.

Palabras clave: Aprendizaje. Laparoscopia. Urologia. Modelos Experimentales.

ABSTRACT

ANIMAL MODELS IN UROLOGICAL LAPAROSCOPIC TRAINING

We present the experience of the Minimally Invasive Surgery Centre (MISC) in the development of a modular training model in laparoscopic surgery. The experience analysis includes the description of the training objectives, the learning process of simple and advance laparoscopic urologic techniques, as well as some current and future considerations before applying the laparoscopic techniques. This learning program pretends to optimize the knowledge of the surgeon and the clinical practice of these surgical techniques. The phases of the learning process have been classified in four levels, which include different modules and models and whose application will depend on the experience and surgical skills. This pyramidal training system permits the student to advance step by step through each level depending on her surgical skills.

We have presented our experience in twelve courses about laparoscopic urology and four courses of laparoscopic radical prostatectomy, in which more than 300 urologists have assisted. Furthermore, some Spanish Urology Units have been developing special experimental training programs on laparoscopic radical prostatectomy, partial nephrectomy or laparoscopic dismembered pyeloplasty with Anderson-Hynes technique.

It has been previously described that laparoscopic modular learning constitutes a very useful concept to avoid problems related to an incomplete and incorrect learning process. Also it seems clear that the laparoscopic training reduces the learning curve in laparoscopic urologic techniques.

Keywords: Learning, Laparoscopy, Urology, Experimental Models. 
$\mathrm{E}$ n los últimos años la cirugía urológica ha sufrido una serie de cambios relacionados, en gran medida, con el desarrollo de las técnicas quirúrgicas de mínima invasión. Las ventajas de este tipo de técnicas han sido ampliamente descritas y relacionadas, en la mayoría de los trabajos, con un menor tiempo de hospitalización, mejores resultados cosméticos y un postoperatorio más ventajoso para el paciente con respecto al abordaje abdominal clásico. No obstante, se definen como "inconvenientes" de las mismas la necesidad de un aprendizaje óptimo.

El concepto de aprendizaje por módulos que presentamos es utilizado con éxito en los programas de aprendizaje de cirugía laparoscópica urológica $^{1}$. La cirugía laparoscópica ha necesitado el desarrollo de técnicas de aprendizaje rápidas, efectivas y fiables llevadas a cabo, en sus primeras etapas, fuera de los quirófanos. Además de la enseñanza y aprendizaje específicos, se requiere un entrenamiento quirúrgico especializado que es preciso ajustar a la experiencia del cirujano. Las etapas de entrenamiento incorporan desde simuladores físicos, simuladores virtuales, hasta la cirugía experimental -en animales y cadáveres, concluyendo el aprendizaje en pacientes humanos siempre con la supervisión directa de cirujanos expertos. Por último, y una vez iniciado en la práctica laparoscópica, el cirujano debe procurarse una formación continuada tanto teórica como práctica ${ }^{2-6}$.

Los cursos de cirugía laparoscópica realizados en los últimos dieciséis años, en el Centro de Cirugía de Mínima Invasión (CCMI), nos han permitido participar en la formación quirúrgica de más de 300 urólogos españoles y europeos. En este periodo se han celebrado trece Cursos de Cirugía Laparoscópica Urológica y seis Cursos de Prostatectomía Radical Laparoscópica, con dos módulos diferenciados en cada curso, siempre bajo los auspicios de la Asociación Española de Urología (AEU). Además se han realizado más de veinticinco estancias de formación de postgrado específicas en urología para el adiestramiento en técnicas quirúrgicas básicas y avanzadas.

Durante este tiempo han sido protocolizados los pasos del aprendizaje de la cirugía laparoscópica urológica ajustándolos a los niveles de experiencia y destreza con la que cuenta el cirujano.
La secuencia de aprendizaje concuerda con una pirámide que incorpora cuatro niveles que el alumno debe superar hasta alcanzar el vértice, que concluye con la aplicación de la técnica quirúrgica en la práctica clínica. Previamente el alumno debe superar, o en su defecto conocer, los tres niveles inferiores.

Este proceso de formación requiere unos estándares formativos mínimos y una acreditación del programa establecido ${ }^{7} \mathrm{y}$, aunque existe un vacío legal, en otros países y Sociedades Científicas se han publicado algunas recomendaciones referentes al entrenamiento y la acreditación de cirujanos en cirugía laparoscópica y toracoscópica $^{8,9}$, que conviene tener en cuenta.

Finalmente el desarrollo tecnológico ha permitido incorporar nuevas herramientas que intentan rebajar los tiempos y costes de aprendizaje (teleformación, simuladores, robótica, realidad virtud, etc.) ${ }^{10-13}$, y que constituyen algunas lineas de trabajo actuales y futuras.

\section{Modelo piramidal de aprendizaje en cirugia laparoscópica}

El aprendizaje en cirugía laparoscópica urológica se ha diferenciado en diversos niveles de formación, adaptados al grado de experiencia y conocimientos del cirujano. El programa de formación quirúrgica comienza en un nivel básico (Nivel 1), caracterizado por transmitir la información más esencial de la cirugía laparoscópica, referente a equipos e instrumental, facilitando el manejo de este instrumental y realizando las maniobras elementales de coordinación de movimientos en los simuladores quirúrgicos (Fig. 1).

Este modelo ha sido adaptado por el CCMI para el aprendizaje de la cirugía laparoscópica urológica en España.

Este nivel se fundamenta en la realización de ejercicios prácticos organizados en diferentes módulos de aprendizaje (prensión, corte, disección y sutura), siguiendo en cada módulo un especial interés en aspectos relacionados con:

a. Conocimiento y manejo de equipos e instrumental laparoscópico (en cursos básicos)

b. La ergonomía en cirugía laparoscópica.

c. Estudio de la anatomía quirúrgica del modelo animal.

d. Realización de la técnica quirúrgica en el modelo animal. 


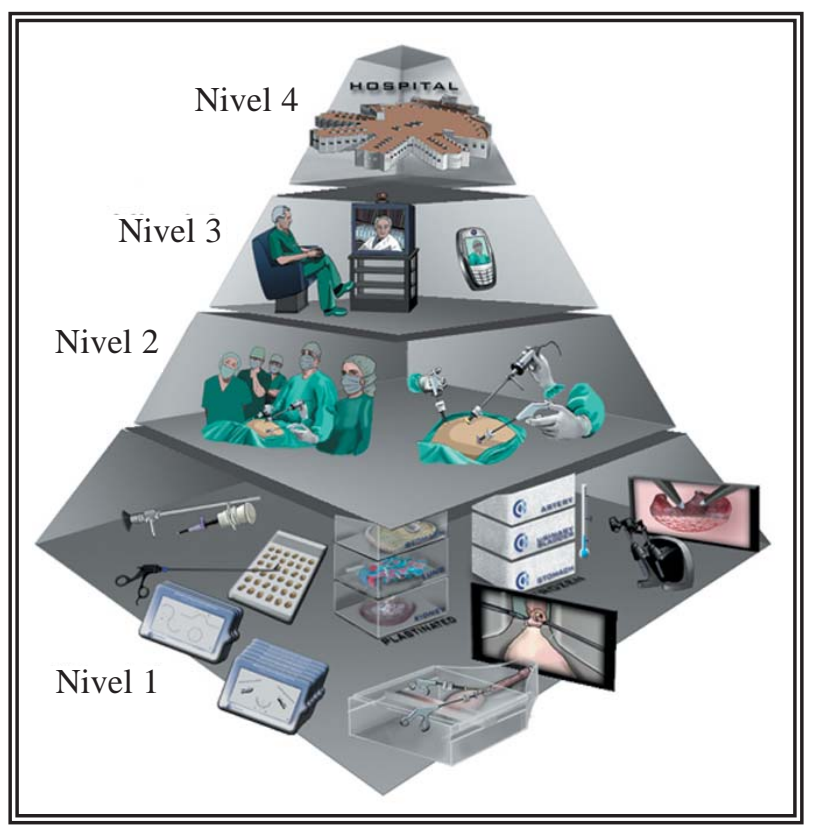

FIGURA 1. Modelo de enseñanza con estructura piramidal donde se diferencian niveles y módulos de aprendizaje.

En un nivel avanzado (Nivel 2), se formaliza el aprendizaje de diferentes técnicas laparoscópicas en animales de experimentación (Fig. 1). El uso de animales vivos minimiza la etapa de formación en pacientes humanos aunque en los últimos años, debido esencialmente al desarrollo tecnológico, se ha favorecido el empleo de simuladores quirúrgicos en urologia ${ }^{14,15}$. No obstante, en el caso de técnicas quirúrgicas avanzadas abogamos por emplear modelos animales con patología de oclusión ureteral, patología renal, desarrollo prostático o modelos de criptorquidia para favorecer un aprendizaje más realista de estas técnicas.

Durante el proceso de aprendizaje quirúrgico también son empleados otros recursos como la teleformación, que constituye una herramienta eficaz para completar el aprendizaje en cirugía laparoscópica. El CCMI aprovecha la red Telesurge ${ }^{10}$ para establecer conexiones entre diferentes usuarios separados geográficamente ${ }^{11}$. Finalmente, en la última fase del aprendizaje, se considera esencial que el alumno en formación aplique los conocimientos adquiridos en especie humana, guiado siempre por un experto en cirugía laparoscópica ${ }^{16}$.

En último término se reconoce por las diferentes sociedades científicas o entidades relacio- nadas con la formación quirúrgica que es primordial la asistencia del cirujano a otros cursos, seminarios, congresos, etc., intentando mantener una formación continuada en cirugía laparoscópica $^{2-4}$.

\section{Principios del aprendizaje laparoscópico. Simulación y ergonomía}

La cirugía laparoscópica presenta algunos inconvenientes derivados, en gran medida, de la manipulación de una gran variedad de instrumentos durante maniobras, más o menos complicadas, a través de la imagen que proporciona un monitor bidimensional. A esta circunstancia hay que unirle la pérdida de sensación táctil y la adopción de posturas forzadas durante periodos de tiempo relativamente largos.

En las fases iniciales los simuladores laparoscópicos inanimados constituyen una herramienta útil para la adquisición de la destreza suficiente en el manejo del nuevo instrumental y ensayar los ejercicios laparoscópicos ${ }^{17}$, bien directamente -a través de su cubierta transparente- o bien con imagen en monitores (Fig. 2), manejando el instrumental con ambas manos, coordinando los movimientos con visión directa.

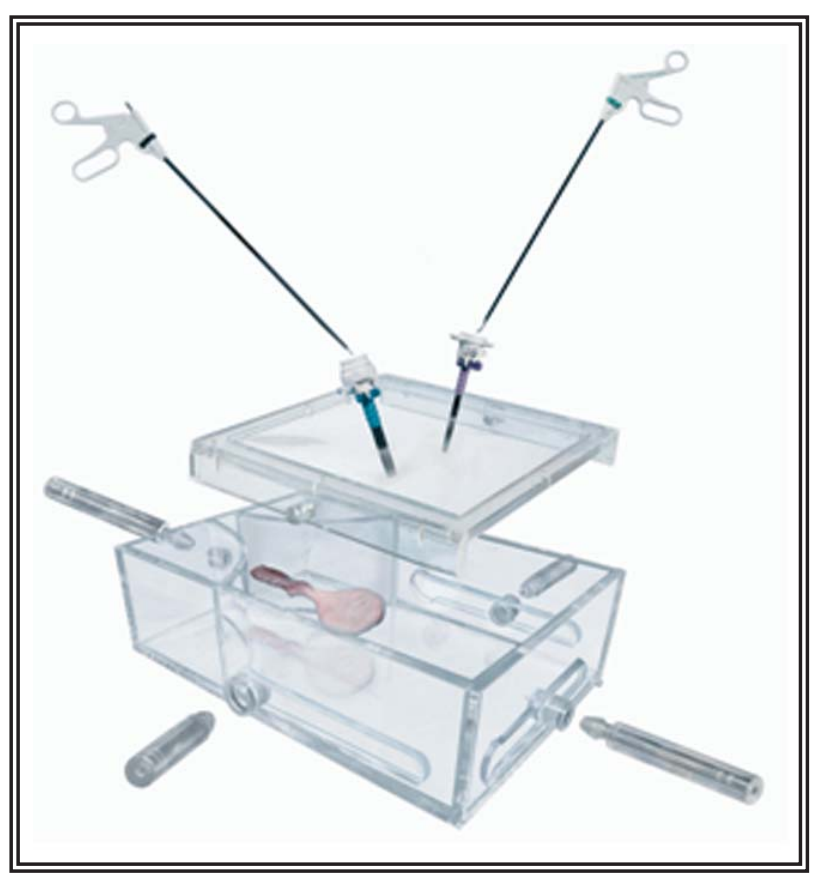

FIGURA 2. Simulador multifuncional y multidisciplinario desarrollado por el CCMI para el entrenamiento en cirugía laparoscópica avanzada. 
Generalmente los ejercicios en el simulador se realizan sobre patrones experimentales como el látex o tejidos orgánicos, siendo muy útiles estos dispositivos para adquirir la habilidad quirúrgica necesaria para efectuar maniobras de mayor complejidad técnica, como la sutura laparoscópica intracorpórea. El dominio de la sutura laparoscópica es inevitable para practicar intervenciones quirúrgicas avanzadas como la prostatectomía radical, nefrectomía parcial o pieloplastia laparoscópicas. Para ello, el siguiente paso en el aprendizaje consiste en llevar a cabo las técnicas, que previamente se han ensayado en el simulador, en piezas de cadáver como estómagos, vejigas de la orina, etc., antes de iniciar los ejercicios en los animales de experimentación ${ }^{2-4}$.

Como ejemplo en el Programa de Formación en Prostatectomía Radical laparoscópica, puesto en marcha en el CCMI en 2003, es empleado un simulador multidisciplinario (Fig. 2) que permite diferenciar distintos grados de dificultad durante la anastomosis uretrovesical ${ }^{4}$, dependiendo en gran medida de la habilidad y experiencia del urólogo.

Por otra parte, en nuestro caso, además de trabajar en el diseño y prestaciones de los simuladores quirúrgicos, llevamos tiempo trabajando en la metodología de la Biomecánica, analizando y evaluando las condiciones de trabajo de los cirujanos en el quirófano, intentando definir las pautas para un uso adecuado del material laparoscópico, fundamentadas siempre en criterios ergonómicos.

Los problemas asociados a la incorrecta manipulación del instrumental y en las anómalas posturas corporales adoptadas pueden provocar la aparición de fatiga muscular (Fig. 3) y en menor medida lesiones músculo-esqueléticas ${ }^{18,19}$. La revisión del entorno quirúrgico nos muestra graves problemas asociados a la cirugía laparoscópica, identificando una serie de criterios ergonómicos que solemos aplicar a la posición que deben adoptar determinadas articulaciones y grupos musculares, quienes participan activamente durante todo el procedimiento laparoscópico ${ }^{20,21}$.

El registro electromiográfico del cirujano muestra una escasa actividad muscular en el cuello y hombro. Sin embargo la imagen de la izquierda, con una postura agachada del cirujano, la electromiografía muestra la aparición de fatiga muscular (Fig. 3).

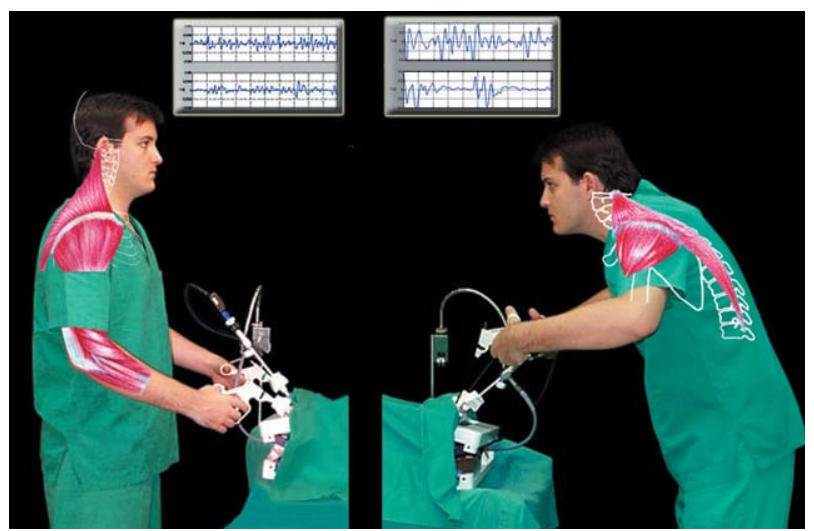

FIGURA 3. La imagen de la izquierda muestra la posición corporal neutral, en bipedestación.

\section{Formación laparoscópica avanzada. Modelos animales en urología}

Las técnicas laparoscópicas urológicas en los animales de experimentación deben acogerse a las normas internacionales y los acuerdos del Consejo de Europa sobre protección de los animales empleados en experimentación animal y otros fines científicos, así como la reciente legislación nacional vigente (Directiva 86/609/CEE y R.D. 1201/2005).

La especie animal que con mayor frecuencia se utiliza como modelo experimental en laparoscopia urológica es la especie porcina, debido esencialmente a las semejanzas anatómicas del aparato urinario con el ser humano ${ }^{22}$ (similar tamaño del riñón, riñón multipapilar, uréteres de similar longitud). Los cerdos que se emplean suelen oscilar entre 25-40 Kg de peso.

El CCMI ha diseñado protocolos laparoscópicos estandarizados, tanto por sistemas orgánicos como por órganos, que son estudiados antes de comenzar la intervención, de tal forma que el alumno se iniciará en las técnicas laparoscópicas más sencillas para continuar con las más avanzadas. En los Cursos de Formación de Laparoscopia Urológica del CCMI se realizan distintos procedimientos en la especie porcina, tales como cistotomía, cirugía del uréter, cirugía vesical, orquidectomía, nefrectomía total, nefrectomía parcial, disección de vasos iliacos, pieloplastia o prostatectomía.

Estamos de acuerdo con otras Sociedades Científicas, como la Asociación Americana de Urologia $^{23}$, en que es evidente el papel que desempeñan los cursos prácticos en la expansión de la cirugía laparoscópica, habiéndose descrito 
la utilidad de un entrenamiento planificado para que los cirujanos dominen la sutura laparoscópica antes de su aplicación clínica en procedimientos avanzados ${ }^{24}$. No obstante, y a pesar de que la cirugia laparoscópica se extiende rápidamente por Europa, el entrenamiento laparoscópico sigue siendo una pieza clave en el desarrollo a medio plazo de este tipo de técnicas ${ }^{25}$.

\section{Protocolo experimental}

Recuerdo Anatómico

Cuando se emplean especies animales durante la formación, como paso previo a la cirugía, es preciso estudiar las particularidades anatómicas de la especie elegida, que en nuestro caso será la porcina. Así, en el cerdo los riñones son parcialmente retroperitoneales, principalmente el riñón derecho. El riñón izquierdo se encuentra más protegido por el peritoneo parietal y ambos se encuentran cubiertos por cantidades variables de grasa perirrenal. El aporte sanguíneo procede de la arteria renal, que puede ser doble, sobre todo en el lado izquierdo, siendo la vena única en ambos lados (Fig. 4).

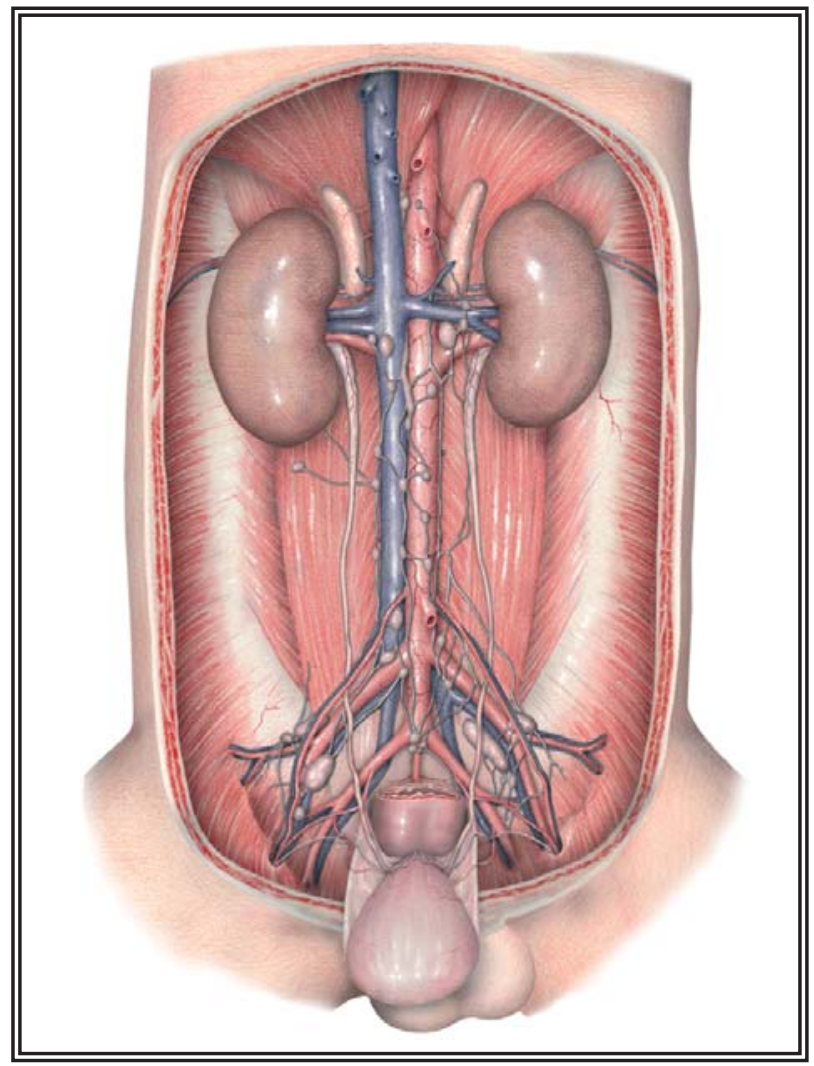

FIGURA 4. Imagen ventral del abdomen porcino con el detalle de los principales trayectos vasculares.
El uréter originado del hilio renal circula en sentido caudal, siendo identificado por su gruesa pared muscular y trayecto retroperitoneal. En su porción abdominal se relaciona con el músculo psoas, la vena cava o la arteria aorta (según sea derecho o izquierdo), continuándose como porción iliaca y concluye como uréter pelviano al cruzarse con los vasos iliacos, para buscar en sentido ventromedial, la cara dorsal de la vejiga, cuya pared perfora en sentido oblicuo (Fig. 4).

\section{Técnica quirúrgica}

Antes de la cirugía el animal es sometido a anestesia general y situado sobre la mesa quirúrgica, procediendo a obtenerse el neumoperitoneo insuflando $\mathrm{CO}_{2}$ a través de la aguja de Veress o por un trocar de Hasson (técnica abierta).

En el supuesto de acceder al riñón y uréter derechos, el cirujano y su ayudante se situarán frente al abdomen del paciente, a uno de los lados de la mesa quirúrgica, de forma que los monitores quedan colocados justo enfrente de ellos y el instrumentista se sitúa al lado del cirujano (Fig. 4). Seguidamente se emplaza un trocar de $10 \mathrm{~mm}$ dorsal a la cicatriz umbilical para el manejo de la óptica. Otros dos o tres trocares, con posición subcostal, en fosa iliaca y en el flanco abdominal son introducidos para completar las intervenciones con abordaje renal y ureteral (Fig. 5).

Tras finalizar la exploración de la cavidad abdominal el riñón es localizado, diferenciándose la arteria renal, vena renal y el uréter en su entrada al riñón. La disección en el área del hilio renal permite identificar los vasos renales; la arteria renal se distingue por su consistencia y

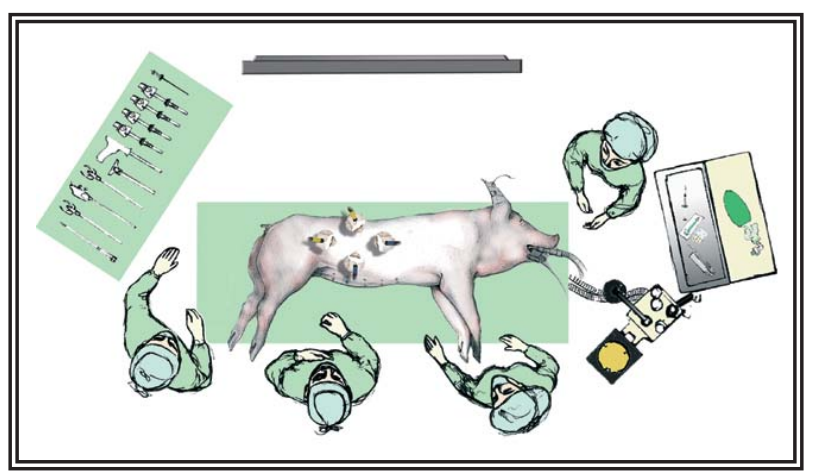

FIGURA 5. Situación del equipo quirúrgico y disposición de los trocares en la técnica de nefrectomia parcial. 
pulso, de forma que la intervención se inicia con la apertura del peritoneo posterior. Mediante unas pinzas o el aspirador se bascula cranealmente el riñón y tras disecar la arteria renal se coloca un torniquete de Rimel. Seguidamente se secciona el parénquima renal sin emplear electrocoagulación (Fig. 6).

Tras la oclusión arterial se secciona el parénquima renal y se completa la hemostasia del lecho de la nefrectomía mediante puntos dobles o simples que apoyamos sobre material hemostático, tipo Surgicel ${ }^{\circledR}$ (Fig. 7). Se consigue una adecuada hemostasia aplicando tres o cuatro puntos de sutura. Finalmente el torniquete es retirado (Fig. 8).

En el caso de la pieloplastia laparoscópica, tras la apertura del peritoneo posterior, se diseca el uréter, la pelvis renal y el polo caudal del riñón. La confección de un punto de sutura sobre la pelvis renal facilitará la manipulación del uréter (Fig. 8). Tras la sutura de la cara posterior de la anastomosis se introduce un catéter doble $\mathrm{J}$ y se realiza la sutura de la cara anterior (Fig. 9). Finalmente se realiza la sutura de la pelvis redundante (Fig. 10).

Como complemento a la formación experimental es recomendable utilizar cadáveres debi-

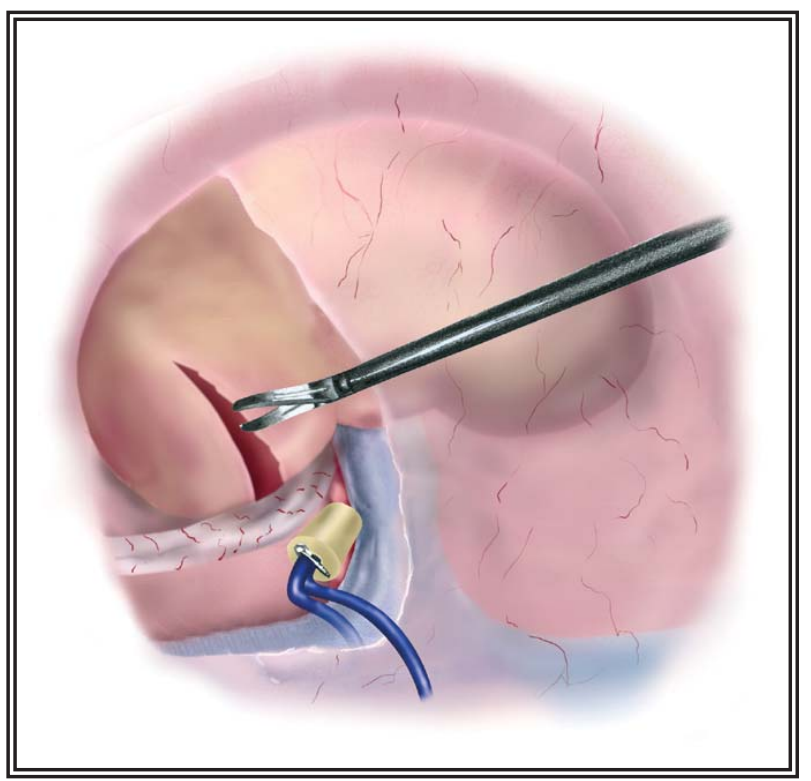

FIGURA 6. La intervención se inicia con la apertura del peritoneo posterior, basculando el riñón con el aspirador y colocando un torniquete alrededor de la arteria renal antes de proceder a la exéresis del parénquima renal.

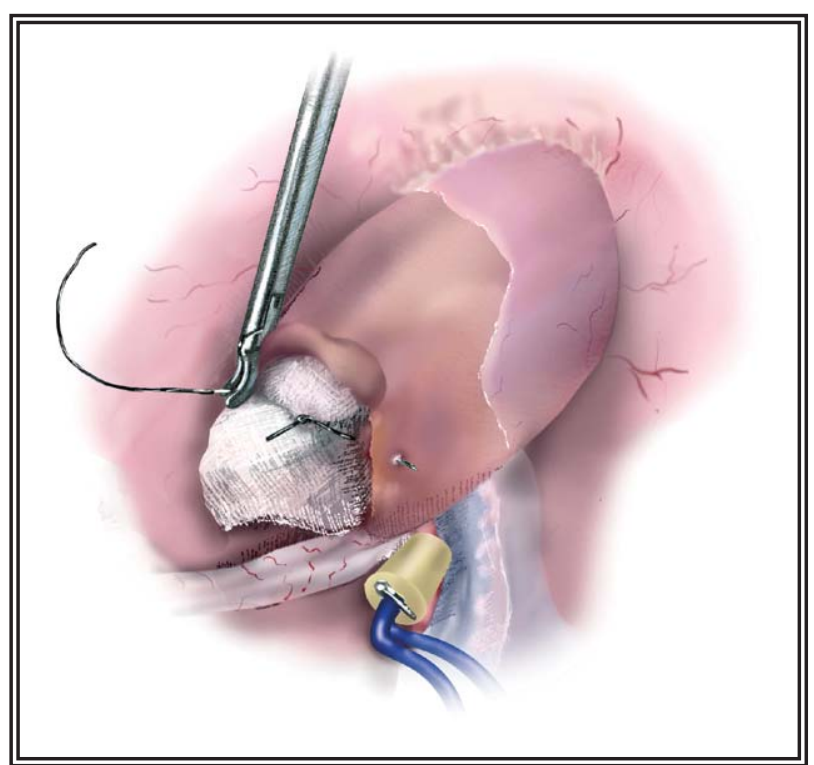

FIGURA 7. Tras la exéresis polar se practica la hemostasia del lecho de la nefrectomia.

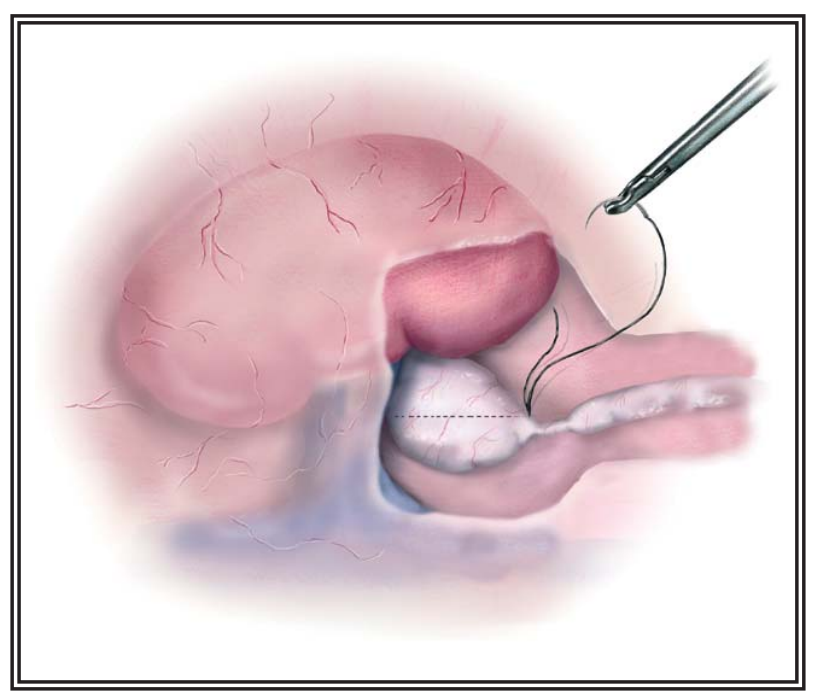

FIGURA 8. Tras realizar una apertura amplia del peritoneo posterior se disecan el uréter, la pelvis renal y el polo caudal del riñón. Las líneas delimitan la zona de sección de la pelvis y la unión pieloureteral. La elaboración de un punto transfixiante favorecerá la ejecución de la sutura.

damente preparados para permitir su empleo con un abordaje laparoscópico ${ }^{26}$. Así, en el CCMI se dispone de cadáveres humanos y animales preparados con la técnica de Thiel $^{27}$, que mantienen un adecuado grado de flexibilidad en las extremidades y conservan una consistencia, color y textura de los órganos internos muy próxima a la real. 


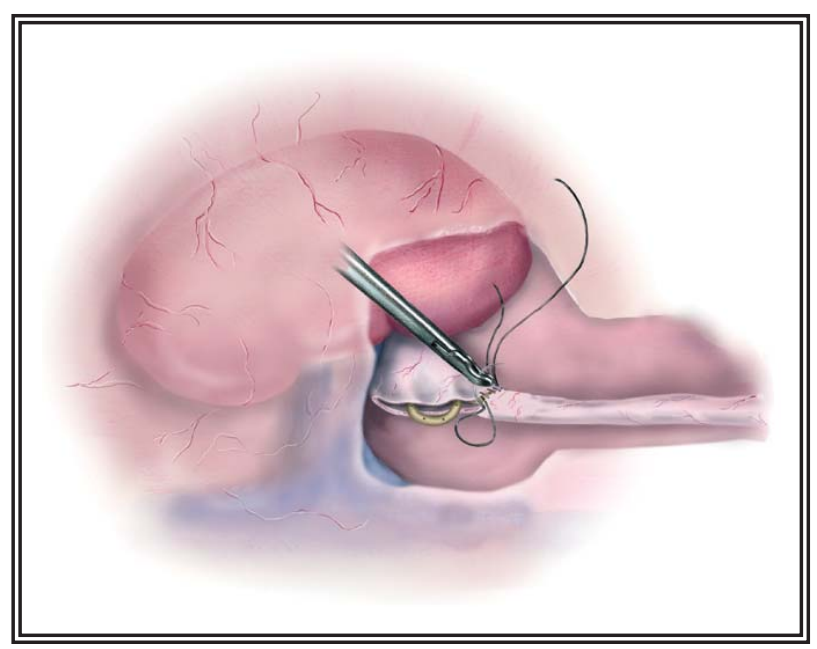

FIGURA 9. Detalle de la inserción del catéter doble $J$ y la realización de la hemisutura de la cara anterior.

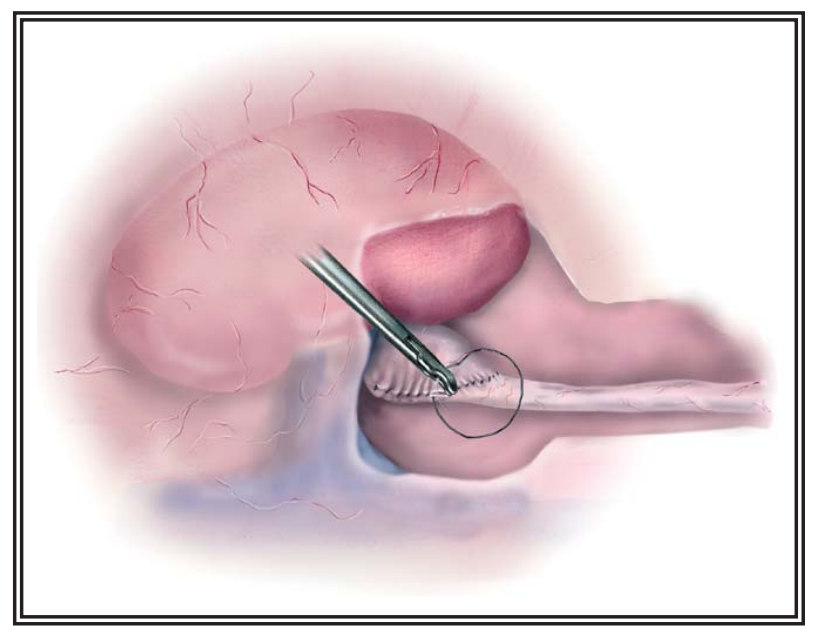

FIGURA 10. Finalizada la sutura ureteral se completa el resto de la sutura de los bordes de la pelvis.

Igualmente, durante el periodo de aprendizaje quirúrgico, se pone a disposición de los alumnos las actuales herramientas de teleformación, que sirven de complemento durante el aprendizaje laparoscópico (Nivel 3).

En una última fase de aprendizaje, o Nivel 4, el alumno se encuentra en disposición de aplicar los conocimientos adquiridos siempre con la supervisión de un experto en cirugía laparoscópica ${ }^{16}$. No obstante, consideramos que el cirujano no debe perder el interés en los avances quirúrgicos y asistir, con relativa frecuencia, a demostraciones prácticas de otros cirujanos más expertos, reuniones científicas, congresos, etc., y procurar mantener una formación continua en cirugía laparoscópica ${ }^{2-4}$.

\section{CONCLUSIONES}

En los últimos años un importante grupo de urólogos españoles ha aprovechado el programa de aprendizaje desarrollado en el CCMI, siempre bajo los auspicios de la Asociación Española de Urología. La experiencia adquirida en este periodo nos muestra que es necesario implantar programas modulares, como el que aquí presentamos, para que el periodo de formación quirúrgica por el que pasa el alumno pueda ser útil y con una futura aplicación clínica.

Podemos concluir que la etapa de formación quirúrgica con simuladores es esencial antes de realizar el aprendizaje de técnicas avanzadas, como la prostatectomía radical, nefrectomía parcial o pieloplastia, que precisan del dominio de las maniobras de sutura laparoscópica intracorpórea. Asimismo el aprovechamiento de las nuevas tecnologías, el desarrollo de modelos animales útiles y las posibilidades de acceder a programas formativos contrastados ponen a disposición del urólogo todas las herramientas necesarias para favorecer la implantación de este tipo de novedosas técnicas en la práctica clínica.

\section{REFERENCIAS}

1. Stolzenburg JU, Schwaibold H, Bhanot SM, Rabenalt R, Do M, Truss M, et al. Modular surgical training for endoscopic extraperitoneal radical prostatectomy. BJU Int. 2005;96(7): 1022-1027.

2. Usón J, Pascual S, Sánchez FM, Hernández FJ. Pautas para el aprendizaje en suturas laparoscópicas. En: Usón J, Pascual S (eds.), Aprendizaje en suturas laparoscópicas, Librería General S.A., Zaragoza, capítulo 2. 1999;38-54.

3. Usón J, Pascual S, Sánchez FM. Aprendizaje y formación en cirugía laparoscópica. En: Bilbao E, Pascual S. Cirugía laparoscópica del Reflujo Gastroesofáfico. Técnica de Nissen. Editorial Centro de Cirugía de Mínima Invasión. Cáceres. 2002; 37-61.

4. Usón J, Pascual S, Sánchez FM, Tejonero MC. Enseñanza de la laparoscopia: Bases prácticas. En: Usón J, Sánchez FM, Roca A, Passas J, Van Velthoven R. Prostatectomía radical laparoscópica. Editorial Centro de Cirugía de Mínima Invasión. Cáceres. 2003; 97-124.

5. Bannenberg J, Meijer D. Setting up and Running Courses. En: Ballantyne GH, Leahy PF, Modlin IM (ed). Laparoscopic surgery. Section VIII: Postdoctoral training for laparoscopic surgery. Chapter 61. W.B. Saunders Co. Philadelphia. 1994; 677-685.

6. Shalhav AL, Dabagia MD, Wagner TT, Koch MO, Lingeman JE. Training postgraduate urologists in laparoscopic surgery: the current challenge. J Urol. 2002;167(5):2135-2137.

7. D’Amico TA, Schwartz LB, Eubanks S. Laparoscopic instrumentation and basic techniques. In: Pappas TN, Schwartz LB, Eubanks S (eds). Atlas of laparoscopic surgery. Chapter 1. Current Medicine. Philadelphia. 1-10. 1996. 
8. Guidelines for granting of privileges for laparoscopic and/or thoracoscopic general surgery. Society of American Gastrointestinal Endoscopic Surgeons (SAGES). Surg Endosc. 1998;12(4):379-380.

9. Hammond L, Ketchum J, Schwartz BF. Accreditation council on graduate medical education technical skills competency compliance: urologic surgical skills. J Am Coll Surg. 2005;201(3):454-457.

10. Masero V, Sánchez FM, Usón J. An integrated system of telemedicine for minimally invasive surgery. J Telemed Telecare. 2000;6 Suppl 2:S88-S89.

11. Masero V, Sánchez FM, Usón J. A telemedicine system for enabling teaching activities. J Telemed Telecare. 2000; 6 Suppl 2:S86-S88.

12. Laguna M, Pilar A, Hatzinger M, Rassweiler J. Simulators and endourological training. Current Opinion in Urology. 2002;12(3):209-215.

13. Usón J, Sánchez FM. El futuro de la cirugía de mínima invasión. Cirugía Mayor Ambulatoria. 1998;3(4):14-18.

14. Shah J, Mackay S, Vale J, Darzi A. Simulation in urologya role for virtual reality?. BJU Int. 2001;88(7): 661-665.

15. Jordan JA, Gallagher AG, McGuigan J, McGlade K, McClure N. A comparison between randomly alternating imaging, normal laparoscopic imaging, and virtual reality training in laparoscopic psychomotor skill acquisition. Am J Surg. 2000;180:208-211.

16. Traxer O, Gettman MT, Napper CA, Scott DJ, Jones DB, Roehrborn CG, Pearle MS et al. The impact of intense laparoscopic skills training on the operative performance of urology residents. J Urol. 2001;166(5):1658-1661.

17. Fried GM, Derossis AM, Bothwell J, Sigman HH. Comparison of laparoscopic performance in vivo with performance measured in a laparoscopic simulator. Surg Endosc. 1999;13:1077-1082.

18. Berguer R. Surgical technology and the ergonomics of laparoscopic instruments. Surg Endosc. 1998;12(5):458-452.

19. Berguer R, Forkey DL, Smith WD. Ergonomics problems associated with laparoscopic surgery. Surg Endosc. 1999; 13(5):466-468.
20. Hemal AK, Srinivas M, Charles AR. Ergonomic problems associated with laparoscopy. J Endourol. 2001;15(5):499503.

21. Berguer R, Rab GT, Abu-Ghaida H, Alarcon A, Chung J. A comparison of surgeons' posture during laparoscopic and open surgical procedures. Surg Endosc. 1997;11(2):139142.

22. Pereira-Sampaio MA, Favorito LA, Sampaio FJ. Pig kidney: anatomical relationship between the intrarenal arteries and the kidney collecting system. Applied study for urological research and surgical training. J Urol. 2004;172(5 Pt 1):2077-2081.

23. Pareek G, Hedican SP, Bishoff JT, Shichman SJ, Wolf JS, Nakada SY. Survey from skills-based hands on learning courses demonstrates increased laparoscopic caseload and clinical laparoscopic suturing. Urology. 2005;66(2): 271-273.

24. Gupta R, Cathelineau X, Rozet F, Vallancien G. Feedback from operative performance to improve training program of laparoscopic radical prostatectomy. J Endourol. 2004;18 (9):836-839.

25. Laguna MP, Schreuders LC, Rassweiler JJ, Abbou CC, van Velthoven R, Janetschek G, et al. Development of laparoscopic surgery and training facilities in Europe: results of a survey of the European Society of Uro-Technology (ESUT). Eur Urol. 2005;47(3):346-351.

26. Katz R, Hoznek A., Antiphon P, Van Velthoven R, Delmas $\mathrm{V}$, Abbou CC. Cadaveric versus porcine models in urological laparoscopic training. Urol Int. 2003;71(3):310-315.

27. Thiel W. Supplement to the conservation of an entire cadaver according to W. Thiel. Ann Anat. 2002;84(3):267-269.

Dr. J. Usón Gargallo

Centro de Cirugía de Mínima Invasión

Avda. Universidad, s/n

10071 Cáceres

E-mail: juson@ccmi.es 